\title{
Diversidad de halófitas chilenas: distribución, origen y hábito
}

\section{Diversity of Chilean halophytes: distribution, origin and habit}

\author{
Fabiola Orrego ${ }^{1,3 *}$, Luz María de la Fuente ${ }^{1,3}$, Miguel Gómez ${ }^{2}$ \& Rosanna Ginocchio ${ }^{1,3}$ \\ ${ }^{1}$ Departamento de Ecosistemas y Medio Ambiente, Facultad de Agronomía e Ing. Forestal, Pontificia Universidad Católica de \\ Chile. Avenida Vicuña Mackenna 4860, Santiago, Chile. \\ ²Departamento de Ciencias Vegetales, Facultad de Agronomía e Ing. Forestal, Pontificia Universidad Católica de Chile. \\ Avenida Vicuña Mackenna 4860, Santiago, Chile. \\ ${ }^{3}$ Center of Applied Ecology and Sustainability, Facultad de Ciencias Biológicas, CP 6513677, Universidad Católica de Chile, \\ Santiago, Chile. \\ *florrego@uc.cl
}

\begin{abstract}
RESUMEN
Las especies halófitas son reconocidas por su capacidad de sobrevivir en ambientes salinos. Sin embargo, esta condición varía enormemente entre los diferentes taxones de plantas halófitas, dificultando su correcta clasificación. Actualmente, la base de datos eHALOPH registra las especies halófitas de todo el mundo bajo un único criterio de salinidad. En este trabajo se presenta el primer listado de halófitas en Chile, elaborado a partir de eHALOPH. Los resultados indican una diversidad de 138 especies, distribuidas en 31 familias. Más del 80\% de estas especies son herbáceas y cerca del 55\% son exóticas. La mayor riqueza se encontró en las regiones de Coquimbo y Valparaíso, y la menor en el territorio insular. Estos resultados son una primera aproximación a la diversidad de especies halófitas en Chile. Al mismo tiempo, plantean un desafío para avanzar con su reconocimiento con el fin de proponer posibles estrategias para su conservación y uso.
\end{abstract}

Palabras clave: Halófita, diversidad vegetal, ecosistemas salinos, eHALOPH.

\begin{abstract}
Halophyte species are recognized for their ability to survive in salty environments. However, this condition greatly varies among plant taxons, difficulting their proper classification. Currently, the eHALOPH database registers halophyte species from around the world, under a single salinity criterion. In the present study, the first list of halophyte species present in Chile made from eHALOPH is presented. Results indicate a diversity of 138 species, distributed in 31 families. Over $80 \%$ of species are herbaceous and nearly $55 \%$ are exotic. The largest richness was found in Coquimbo and Valparaíso regions, and the lowest in the insular territory. These results are a first approximation to halophyte diversity in Chile. At the same time, it poses a challenge to advance in their recognition in order to propose strategies for their conservation and use.
\end{abstract}

KeYwords: Halophyte, plant diversity, saline ecosystems, eHALOPH.

\section{INTRODUCCIÓN}

El término halófita (halos $=s a l ;$ phyta $=$ planta de) se utiliza hace más de 200 años para definir a aquellas especies de plantas que han hecho de los ecosistemas salinos su hábitat (Flowers et al. 1986). Sin embargo, la gran diversidad de ambientes en los que existen y la gran cantidad de estrategias que usan para tolerar o evadir el exceso de salinidad en el sustrato, ha derivado en la proliferación de variadas definiciones (Breckle 1990, Grigore et al. 2010). Esto crea una fuente de incertidumbre a la hora de clasificar una especie como halófita, tanto a nivel regional como global (Grigore et al. 2014). A pesar de ello, en la literatura actual se ha llegado a un consenso con respecto al uso de la concentración de cloruro de sodio $(\mathrm{NaCl})$ como el parámetro que permite discriminar a las especies halófitas de aquellas sensibles a la salinidad (Flowers \& Colmer 2008). Sobre la base de esta definición se han creado los primeros listados de especies halófitas a nivel global (Aronson 1989, Menzel \& Lieth 2003, Santos et al. 2015).

En la actualidad, perturbaciones tales como la degradación y salinización de los suelos devuelven el interés hacia este grupo de especies. Sus principales usos están enfocados en el desarrollo de la agricultura biosalina (Koyro 2003, 
Toderich et al. 2008), la rehabilitación de suelos degradados y contaminados con residuos mineros (Al-Farrajii \& Al-Hilli 1994, Aronson \& Le Floc'h 1995, Lutts \& Lefevre 2015), la generación de biomasa para forraje (Lailhacar et al. 1995) y como biofiltro de residuos municipales y aguas residuales (Shpigel et al. 2013, Buhmann et al. 2015). A pesar del gran valor de estas especies, su identificación y clasificación sigue siendo un desafío a nivel local y regional.

En Chile, el estudio de este grupo se ha realizado a través de la descripción de las especies presentes en hábitat salinos (San Martín et al. 1992, Teillier 1998, Teillier \& Becerra 2003, Ramírez \& Álvarez 2012, entre otros) y la evaluación de los rasgos fisiológicos y morfológicos que favorecen la tolerancia a la salinidad (Rhodes \& Felker 1988, Poblete et al. 1991, Bartels \& Dinakar 2013). Dichos resultados indican que nuestro país posee una riqueza importante de especies halófitas; sin embargo, la información sobre la diversidad de estas especies está descrita sólo a nivel local. Por ello, consideramos esencial identificar la diversidad de este grupo de especies a nivel nacional con el fin de generar estrategias de conservación y definir sus potenciales usos.

En este contexto, el objetivo de este trabajo es proponer el primer listado de halófitas vasculares presentes en Chile y señalar sus principales parámetros ecológicos utilizando el listado global de especies eHALOPH.

\section{MATERIALES Y MÉTODOS}

Listado de halófitas PRESENTES EN Chile

Este trabajo utilizó como fuente principal la base de datos eHALOPH. Esta consiste en un registro global de especies halófitas basado en las publicaciones de Aronson (1989) y Menzel \& Lieth (2003), que se mantiene en permanente actualización gracias al aporte de investigadores de todo el mundo (Santos et al. 2015). La definición de halófita que sustenta este listado corresponde a aquellas especies capaces de "tolerar una conductividad eléctrica de al menos $7,8 \mathrm{dSm}^{-1}$ (equivalente a $80 \mathrm{mM}$ de $\mathrm{NaCl}$ ) durante tiempos significativos de su ciclo de vida" (http://www.sussex. ac.uk/affiliates/halophytes 09/11/17). Este listado es de libre acceso y está publicado en la web http://www.sussex.ac.uk/ affiliates/halophytes/.

Para elaborar el listado nacional de halófitas se contrastó el listado eHALOPH (consultado el 13 de diciembre de 2017) con las especies presentes en Chile según el Catálogo de las Plantas Vasculares del Cono Sur (Zuloaga et al. 2008), disponible en la página web http://www2.darwin. edu.ar/. No se consideraron subespecies ni variedades en la elaboración de este listado.

Para cada especie halófita presente en Chile se registró la familia, hábito, origen y distribución regional. Esta información se recopiló a partir del mismo catálogo y el inventario nacional de especies de Chile (MMA 2017).
La distribución regional de las especies se presentó en base a 13 regiones administrativas y tres zonas insulares: Archipiélago de Juan Fernández, Islas Desventuradas e Isla de Pascua. Esta forma de presentación responde a las categorías de distribución utilizada en el Catálogo de Plantas Vasculares del Cono Sur. Adicionalmente, varias publicaciones fueron utilizadas para complementar los datos faltantes (Marticorena \& Quezada 1985, Matthei et al. 1995, Ramírez \& San Martín 2006, Fuentes et al. 2013).

\section{RESULTADOS}

Riqueza específica de las familias de halófitas en Chile El listado de especies halófitas de Chile está compuesto por un total de 138 especies (Anexo 1), distribuidas en 31 familias (Tabla 1). Las familias con mayor riqueza de halófitas son Poaceae con 29 especies, Amaranthaceae con 20, Fabaceae con 11, Asteraceae con 9 y Cyperaceae con 8 (Tabla 1). De las 31 familias registradas, 26 poseen menos de un 5\% de representación. Sólo dos familias tienen más de $10 \%$ de representación: Poaceae y Chenopodiaceae con 21,01 y $14,49 \%$, respectivamente (Tabla 1 ).

Del grupo de las gimnospermas, solo se encontró la familia Ephedraceae, representada por una especie, Ephedra ochreata. Las otras 30 familias pertenecen al grupo de las angiospermas, dentro de las cuales hay 26 familias de dicotiledóneas y 5 familias de monocotiledóneas.

DiSTRIBUCIÓN, ORIGEN Y HÁBITO DE LAS ESPECIES HALÓFITAS DE Chile

La mayor riqueza de especies halófitas se concentra en la zona centro del país, con una disminución progresiva hacia el extremo sur y el territorio insular (Tabla 2). Las regiones de Coquimbo (COQ) y Valparaíso (VAL) tienen la mayor riqueza, con 81 especies de halófitas cada una. Le siguen las regiones del Biobío (BIO) con 74 y la Metropolitana (RME) con 70. Al contrario, las regiones de Aysén (AIS) y de Magallanes y de la Antártica Chilena (MAG) presentan la menor riqueza de especies del territorio continental, con 25 y 37 registros, respectivamente. El territorio insular también presenta una baja riqueza de especies halófitas, siendo las islas Desventuradas la zona de menor número de especies en el territorio nacional (6 especies).

De las 138 especies de halófitas que hay en Chile, 4 son endémicas (3\%), 58 nativas (42\%) y 76 exóticas (55\%) (Tabla 2). Las cuatro especies de halófitas endémicas registradas son Prosopis tamarugo, Atriplex atacamensis, Atriplex repanda y Hordeum brachyantherum, todas presentes en la zona norte y centro de Chile (Anexo 1). En la zona sur, la única especie endémica registrada corresponde a Hordeum brachyantherum, presente en la región de Magallanes y de la Antártica Chilena. En el territorio insular no se registran especies endémicas. 
Tabla 1. Distribución de la riqueza de especies halófitas de Chile según su familia $(n=138)$. Los resultados se presentan en orden alfabético. / Distribution of chilean halophyte species richness by its family $(\mathrm{n}=138)$. Results presented in alphabetical order.

\begin{tabular}{|c|c|c|}
\hline FAMILIA & RiQuezA & $\%$ \\
\hline Aizoaceae & 6 & 4,35 \\
\hline Anacardiaceae & 1 & 0,72 \\
\hline Apiaceae & 2 & 1,45 \\
\hline Araliaceae & 1 & 0,72 \\
\hline Asteraceae & 9 & 6,52 \\
\hline Brassicaceae & 5 & 3,62 \\
\hline Caryophyllaceae & 2 & 1,45 \\
\hline Convolvulaceae & 2 & 1,45 \\
\hline Cyperaceae & 8 & 5,80 \\
\hline Chenopodiaceae & 20 & 14,49 \\
\hline Ephedraceae & 1 & 0,72 \\
\hline Euphorbiaceae & 2 & 1,45 \\
\hline Fabaceae & 11 & 7,97 \\
\hline Frankeniaceae & 2 & 1,45 \\
\hline Goodeniaceae & 1 & 0,72 \\
\hline Heliotropiaceae & 1 & 0,72 \\
\hline Juncaceae & 5 & 3,62 \\
\hline Juncaginaceae & 2 & 1,45 \\
\hline Malvaceae & 2 & 1,45 \\
\hline Plantaginaceae & 7 & 5,07 \\
\hline Plumbaginaceae & 1 & 0,72 \\
\hline Poaceae & 29 & 21,01 \\
\hline Polygonaceae & 3 & 2,17 \\
\hline Portulacaceae & 1 & 0,72 \\
\hline Potamogetonaceae & 3 & 2,17 \\
\hline Primulaceae & 2 & 1,45 \\
\hline Ruppiaceae & 1 & 0,72 \\
\hline Solanaceae & 4 & 2,90 \\
\hline Typhaceae & 2 & 1,45 \\
\hline Verbenaceae & 1 & 0,72 \\
\hline Zygophyllaceae & 1 & 0,72 \\
\hline Riqueza total & 138 & \\
\hline
\end{tabular}

En Chile continental, las regiones del extremo norte (Arica y Parinacota, Tarapacá, Antofagasta y Atacama) apenas superan el $50 \%$ de especies halófitas nativas y endémicas (Tabla 2). Esta tendencia se invierte en las regiones de la zona centro y sur del país, donde el porcentaje de halófitas exóticas supera el 50\%. En los sistemas insulares este número es aún mayor, con valores sobre el $80 \%$.

El hábito predominante entre las halófitas chilenas es el herbáceo, con 114 especies que representan un $82,6 \%$ de la riqueza total de halófitas. Las hierbas perennes son el grupo mayoritario, con el 53\% del total de las especies descritas (Tabla 2). Las especies halófitas leñosas constituyen un grupo menos abundante, con 5 árboles, 13 arbustos y 5 subarbustos, los que en conjunto representan casi el 17\% del total de especies. De las 138 especies registradas sólo existe una especie con hábito de enredadera, Calystegia sepium (Anexo 1).

Con respecto a la distribución regional de los distintos hábitos, las hierbas perennes son el hábito de mayor presencia en todas las regiones del país, con al menos un $40 \%$ de representación en cada una de ellas (Tabla 2). En la zona sur, este valor es aún mayor (60\%).

Las herbáceas anuales corresponden al segundo grupo más abundante después de las herbáceas perennes en todas las regiones y presentan una mayor riqueza en regiones de la zona norte y centro, con una disminución progresiva hacia el sur (Tabla 2).

La mayor frecuencia de especies leñosas (arbóreas, arbustivas y subarbustivas) ocurre en las regiones de Atacama y Coquimbo con 13 y 12 especies, respectivamente (Tabla 2). En cambio, la frecuencia de este grupo de especies disminuye notoriamente hacia el extremo sur y en el territorio insular, con menos de 3 especies.

\section{DISCUSIÓN Y CONCLUSIONES}

RiQUEZA DE FAMILIAS HALÓFITAS

Este es el primer estudio que compila la diversidad de especies halófitas presentes en Chile, a partir de un listado colaborativo mundial. En él se presentan 138 especies de halófitas, distribuidas en 31 familias, lo que equivale a un 2,3\% de las especies y un 16,8\% de las familias de flora vascular descritas para Chile (Marticorena 1990). Las familias más diversas son Poaceae (29 especies), Chenopodiaceae (20 especies), Fabaceae (11 especies), Asteraceae (9 especies) y Cyperaceae (8 especies). Si bien esta representación coincide con algunas de las familias de mayor riqueza en Chile (Asteraceae, Poaceae y Fabaceae) (Comisión Nacional del Medio Ambiente 2008), la familia Chenopodiaceae no aparece en este grupo, a pesar de ser la segunda familia de mayor riqueza de halófitas a nivel nacional (Tabla 1) y la primera a nivel mundial (Santos et al. 2015).

A nivel global, las 138 especies halófitas descritas en Chile representan un 9,3\% de las 1479 especies identificadas por la base de datos eHALOPH (https://www.sussex.ac.uk/ affiliates/halophytes/). Este valor está muy por debajo de las 673 especies nativas y endémicas descritas para Argentina a través de esta misma herramienta (Cantero et al. 2016) o las más de 300 especies descritas a partir de registros monográficos y trabajos de terreno en países de Europa (Grigore 2008) y Asia (Khan \& Qaiser 2006, Ghazanfar et al 2014). La baja riqueza de halófitas a nivel nacional puede ser 
reflejo de la baja diversidad de especies en Chile comparada a la de otros países en Sudamérica (Comisión Nacional del Medio Ambiente 2008); o bien, estar influenciada por la alta presencia de especies de origen euroasiático en los registros de la base de datos eHALOPH.

ORIGEN DE LAS ESPECIES

Un 55\% de las especies halófitas presentes en Chile son exóticas. Del $45 \%$ restante, un $42 \%$ corresponde a especies nativas, y sólo un $3 \%$ a especies endémicas (Tabla 2). Este patrón difiere significativamente al descrito para la flora vascular en Chile, compuesta por más de un $85 \%$ de especies nativas (Fuentes et al. 2013), de las que casi la mitad son endémicas (Moreira-Muñoz 2011). Al respecto, es importante considerar que la base de datos eHALOPH se construyó desde dos publicaciones (Aronson 1989, Menzel $\&$ Lieth 2003), cuyo objetivo fue la recopilación de especies tolerantes a la salinidad con potencial uso económico. En ese sentido, no nos debe sorprender que, al contrastar este listado con la flora de Chile, se encuentre una presencia importante de especies exóticas, o que las familias con mayor diversidad de halófitas también posean la mayor riqueza de especies introducidas (Fuentes et al. 2013, Fuentes et al. 2014). Así también, la distribución multirregional de este grupo de especies (O'Leary \& Glenn 1994) y el escaso conocimiento acerca de halófitas autóctonas en Chile (Ramírez \& Álvarez 2012) puede ser otra causante de los resultados observados.

TABLA 2. Riqueza, hábito y origen de las especies halófitas presentes en Chile según su distribución geográfica. Distribución: TAR= Región de Arica y Parinacota, Región de Tarapacá; $\mathrm{ANT}=$ Región de Antofagasta; $\mathrm{ATA}=$ Región de Atacama; $\mathrm{COQ}=\mathrm{Región}$ de Coquimbo; $\mathrm{VAL}=$ Región de Valparaíso; RME= Región Metropolitana; LBO= Región del Libertador General Bernardo O’Higgins; MAU= Región del Maule; $\mathrm{BIO}=$ Región del Biobío; ARA= Región de la Araucanía; LLA= Región de Los Ríos y Región de Los Lagos; AIS= Región de Aysén del General Carlos Ibáñez del Campo; MAG= Región de Magallanes y Antártica Chilena; IDE= Islas Desventuradas; IPA= Isla de Pascua; JFE= Archipiélago de Juan Fernández. Origen: EN= endémica; NA= nativa; EX= exótica. Forma de vida: HA= herbácea anual; HAB= herbácea anual o bianual; $\mathrm{HP}=$ herbácea perenne; $\mathrm{Ar}=$ arbustiva; $\mathrm{Sa}=$ subarbustiva; $\mathrm{Ab}=$ arbórea; $\mathrm{E}=$ enredadera perenne / Richness, habit and origin of halophyte species present in Chile by geographical distribution. Distribution: TAR=Arica y Parinacota Region, Tarapacá Region; ANT= Antofagasta Region; $\mathrm{ATA}=$ Atacama Region; $\mathrm{COQ}=$ Coquimbo Region; $\mathrm{VAL}=$ Valparaíso Region; $\mathrm{RME}=\mathrm{Metropolitan} \mathrm{Region}$; $\mathrm{LBO}=$ Libertador Bernardo O'Higgins Region; MAU= Maule Region; BIO=Biobío Region; ARA=Araucanía Region; LLA=Los Ríos Region and Los Lagos Region; AIS= Aysén del General Carlos Ibáñez del Campo Region; MAG= Magallanes y Antártica Chilena Region; IDE= Islas Desventuradas; IPA= Easter Island; JFE= Archipiélago de Juan Fernández. Origin: EN= endemic; NA= native; EX= exotic. Life form: HA= anual herb; $\mathrm{HAB}=$ anual or bianual herb; $\mathrm{HP}=$ perennial herb; $\mathrm{Ar}=$ shrub; $\mathrm{Sa}=$ subshrub; $\mathrm{Ab}=$ tree; $\mathrm{E}=$ perennial vine.

\begin{tabular}{|c|c|c|c|c|c|c|c|c|c|c|c|c|}
\hline & \multirow[t]{2}{*}{ REGIÓN } & \multirow[t]{2}{*}{ RiQUeZA } & \multicolumn{7}{|c|}{ HÁBITO ( $\mathrm{N}^{\circ}$ ESPECIES) } & \multicolumn{3}{|c|}{ ORIGEN ( $\mathrm{N}^{\circ}$ ESPECIES $)$} \\
\hline \multirow{5}{*}{ 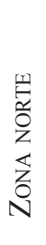 } & & & HA & $\mathrm{HP}$ & $\mathrm{Hab}$ & $\mathrm{Ab}$ & $\mathrm{Ar}$ & $\mathrm{Sa}$ & $\mathrm{E}$ & EN & NA & EX \\
\hline & TAR & 49 & 11 & 28 & 1 & 2 & 4 & 3 & 0 & 2 & 25 & 22 \\
\hline & ANT & 58 & 13 & 32 & 4 & 2 & 3 & 4 & 0 & 2 & 29 & 27 \\
\hline & ATA & 61 & 15 & 30 & 3 & 2 & 8 & 3 & 0 & 2 & 30 & 29 \\
\hline & COQ & 81 & 24 & 42 & 3 & 2 & 6 & 4 & 0 & 2 & 36 & 43 \\
\hline \multirow{5}{*}{ 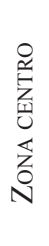 } & VAL & 81 & 23 & 46 & 4 & 2 & 3 & 2 & 1 & 1 & 32 & 48 \\
\hline & RME & 70 & 19 & 40 & 4 & 2 & 3 & 1 & 1 & 1 & 29 & 40 \\
\hline & LBO & 44 & 15 & 18 & 4 & 2 & 3 & 2 & 0 & 0 & 18 & 26 \\
\hline & MAU & 59 & 18 & 34 & 3 & 1 & 2 & 1 & 0 & 1 & 23 & 35 \\
\hline & $\mathrm{BIO}$ & 74 & 24 & 39 & 4 & 1 & 3 & 2 & 1 & 0 & 27 & 47 \\
\hline \multirow{4}{*}{ 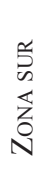 } & ARA & 57 & 17 & 33 & 3 & 1 & 1 & 1 & 1 & 0 & 20 & 37 \\
\hline & LLA & 58 & 15 & 36 & 4 & 0 & 1 & 1 & 1 & 0 & 24 & 34 \\
\hline & AIS & 25 & 5 & 16 & 2 & 1 & 0 & 0 & 1 & 0 & 12 & 13 \\
\hline & MAG & 37 & 6 & 25 & 3 & 0 & 2 & 1 & 0 & 1 & 17 & 19 \\
\hline \multirow{5}{*}{$\underset{\omega}{\infty}$} & IDE & 6 & 2 & 3 & 0 & 0 & 1 & 0 & 0 & 0 & 0 & 6 \\
\hline & JFE & 26 & 10 & 12 & 2 & 1 & 0 & 1 & 0 & 0 & 3 & 23 \\
\hline & IPA & 13 & 2 & 9 & 0 & 1 & 0 & 0 & 1 & 0 & 2 & 11 \\
\hline & Total Chile & 138 & 36 & 73 & 5 & 5 & 13 & 5 & 1 & 4 & 58 & 76 \\
\hline & $\%$ Total Chile & 100 & 26,1 & 52,9 & 3,6 & 3,6 & 9,4 & 3,6 & 0,7 & 2,9 & 42 & 55,1 \\
\hline
\end{tabular}


HÁBITO DE LAS ESPECIES HALÓfITAS PRESENTES EN CHILE

Más del $80 \%$ de las especies halófitas identificadas en Chile son herbáceas. Este grupo, además, presenta la mayor riqueza en todas las regiones del país, incluso en el territorio insular (Tabla 2). Esta predominancia es llamativa, pues a nivel global se ha descrito que la relación de riqueza entre halófitas leñosas y herbáceas es más bien equitativa (Aronson 1989, Khan \& Qaiser 2006, Ghazanfar et al. 2014). La alta representación de este grupo en Chile se puede explicar por la presencia de ciertos tipos de hábitat que favorecen la colonización de hierbas tolerantes a la salinidad, tales como ecosistemas costeros, humedales y lagunas salobres (San Martín et al. 1992, Teillier \& Becerra 2003, Ramírez \& Álvarez 2012). Al mismo tiempo, la alta proporción de especies exóticas en el grupo de hierbas halófitas puede ser un reflejo de su éxito en el uso de hábitat naturales y perturbados, tal como lo ha descrito Ramírez et al. (2018) en marismas de la Región del Biobío.

Por otra parte, las halófitas leñosas en Chile apenas representan un $16,6 \%$ del listado total. Pero, al contrario de las herbáceas, la mayoría de estas $(82,6 \%)$ son nativas o endémicas de las zonas norte y central (Tabla 2). La riqueza de especies leñosas en la zona norte se explica principalmente por la presencia de géneros nativos con dos o más especies (Atriplex, Prosopis, Lycium, Frankenia y Suaeda), cuya distribución regional se encuentra más bien restringida al territorio entre las regiones de Arica y Parinacota y Coquimbo (Anexo 1). En el norte aparecen también tres de los cuatro árboles endémicos o nativos presentes en Chile: Prosopis tamarugo, P. chilensis y Geoffroea decorticans. Al respecto, se ha descrito que la condición de halofitismo entre especies arbóreas es rara (O’Leary \& Glenn 1994). Aún más, si se considera que gran parte de los árboles halófitos descritos a nivel global crecen en manglares, y ecosistemas de interfaz marino-terrestre, sistemas radicalmente distintos a los que alojan a este grupo de especies en Chile.

Al igual que las especies arbóreas, la diversidad de arbustos halófitos es bastante baja. Sin embargo, con una riqueza de 18 especies, 15 de las cuales son nativas y/o endémicas, vale la pena resaltar el componente autóctono de este grupo. Estas especies están representadas por variantes nativas y endémicas de géneros con amplia distribución mundial, como Lycium, Atriplex, Suaeda y Senecio. Esto podría indicar que, dentro de la gran diversidad de estos géneros, ocurre cierto nivel de adaptación local a ecosistemas salinos.

\section{Perspectivas para el estudio de halófitas en Chile}

Hoy en día existe amplio conocimiento acerca de la fisiología y las estrategias de adaptación que utilizan las especies halófitas para enfrentarse a distintos sistemas salinos (Munns \& Tester 2008). Sin embargo, existe un gran vacío de información con respecto a su diversidad a nivel nacional y a las distintas estrategias que utilizan para adaptarse a este tipo de ambientes en Chile.

Al realizar una búsqueda online de libros y artículos científicos con las palabras "halófita" y "Chile", se encuentran listados de especies que existen en hábitats salinos, análisis paleobotánicos de ecosistemas antiguos, estudios de acumulación y tolerancia a metaloides y estudios acerca del uso de halófitas para la nutrición humana y animal. La mayoría de estos artículos fueron publicados entre 1980 y 2000 y denotan la falta de información sistematizada sobre la riqueza de especies halófitas presentes en Chile y de sus características ecológicas. En este contexto, el presente listado, realizado a partir de los registros obtenidos en la base de datos eHALOPH, se constituye como una primera aproximación para compilar la diversidad de las especies halófitas presentes en Chile. Sin embargo, el enriquecimiento de este listado, ya sea a través de una exhaustiva revisión de fuentes bibliográficas nacionales, y la generación de nuevos aportes a dicha base de datos, requiere de la activa colaboración de investigadores y académicos. De este modo, identificar nuevas especies halófitas y ampliar nuestro conocimiento ecológico acerca de ellas nos permitirá también identificar nuevas oportunidades para relevar la importancia de esta parte del patrimonio natural a través de su uso en diferentes áreas.

\section{AGRADECIMIENTOS}

Proyecto CONICYT FB-0002-2014 (Center of Applied Ecology and Sustainability, CAPES UC). Beca CONICYT doctorado 21141059.

\section{REFERENCIAS}

Al-FarrajiI, F., Al-Hilli, M.R. 1994. Halophytes and desertification control in Iraq. In: Squires V.R., Ayoub A.T (eds.), Halophytes as a resource for livestock and for rehabilitation of degraded lands, pp. 239-248. Springer, Switzerland.

Aronson, J.A. 1989. Salt Tolerant Plants of the World. Office of Arid Land Studies, University of Arizona. Arizona, U.S.A. 77 pp.

Aronson, J., Le Floc'H, E. 1995. Restoration ecology of saltaffected, arid and semi-arid lands. In: Choukr-AllAh (ed.), Halophytes and biosaline agriculture, pp. 55-71. 53. CRC Press, U.S.A.

Bartels, D., Dinakar, C. 2013. Balancing salinity stress responses in halophytes and non-halophytes: A comparison between Thellungiella and Arabidopsis thaliana. Functional Plant Biology 40: 819-831.

Breckle, S. 1990. Salinity tolerance of different halophyte types. In: El Bassam, N., Dambroth, M., Loughman, B.C. (eds.), Genetic Aspects of Plant Mineral Nutrition. Developments in Plant and Soil Sciences, Vol. 42, pp. 167-175. Springer, Dordrecht, Netherlands.

Buhmann, A.K., Waller, U., Wecker, B., Papenbrock, J. 2015. Optimization of culturing conditions and selection of species for the use of halophytes as biofilter for nutrient- 
rich saline water. Agricultural Water Management 149: 102-114.

Cantero, J.J., Palchetti, V., NúÑez, C., Barboza, G. 2016. Halophytic flora of Argentina: a checklist and an analysis of its diversity. In: Khan, M.A., Boër, B., Özturk, M., Clüsener-Godt, M., Gul, B., Breckle, S.-W. (eds.), Sabkha ecosystems, pp. 137-204. Springer, Switzerland.

Comisión Nacional del Medio Ambiente. 2008. Biodiversidad de Chile, Patrimonio y Desafíos. Editorial Ocho Libros, Santiago, Chile. 640 pp.

Flowers, T.J., Colmer, T.D. 2008. Salinity tolerance in halophytes. New Phytologist 179(4): 945-963.

Flowers, T.J., Hajtbagheri, M., Clopson, N.J.W. 1986. Halophytes. The Quarterly Review of Biology 61(3): 313-337.

Fuentes, N., Pauchard, A., SÁnchez, P., Esquivel, J., Marticorena, A. 2013. A new comprehensive database of alien plant species in Chile based on herbarium records. Biological Invasions 15(4): 847-858.

Fuentes, N., SÁnchez, P., Pauchard, A., Urrutia, J., Cavieres, L., Marticorena, A. 2014. Plantas invasoras del centro-sur de Chile: una guía de campo. Laboratorio de Invasiones Biológicas (LIB), Concepción, Chile. 276 pp.

Ghazanfar, S.A., Altundag, E., Yaprak, A.E., Osborne, J., Tug, G.N., Vural, M. 2014. Halophytes of Southwest Asia. In: Khan, M.A., Böer, B., Öztürk, M., Al Abdessalaam, T.Z., Clüsener-Godt, M., Gul, B. (eds), Sabkha Ecosystems, Vol 4: Cash Crop Halophyte and Biodiversity Conservation, pp. 105-133. Springer, Dordrecht, The Netherlands.

Grigore, M. 2008. Halophytotaxonomy: list of romanian salt tolerant plants. PIM, Iasi. $137 \mathrm{pp}$.

Grigore, M., Ivanescu, L., Toma, C. 2014. Halophytes and their habitats: finding a place within plant ecological classes. In: Grigore, M., Ivanescu, L., Toma, C., Halophytes: an integrative anatomical study, pp. 27-31. Springer, Cham.

Grigore, M., Toma, C., Boscaiu, M. 2010. Dealing with halophytes: an old problem, the same continuous exciting challenge. Analele Stiintifice ale Universitatii “Al.I. Cuza" din Iasi 56(1): 21-32.

KHAN, M., QAISER, M. 2006. Halophytes of Pakistan: characteristics, distribution and potential economic usages. In: Khan, M.A., Böer, B., Kust, G.S., Barth, H.J. (eds.), Sabkha Ecosystems, Vol 2: West and Central Asia, pp. 129-153. Springer, Dordrecht, The Netherlands.

Koyro, H.W. 2003. Study of potential cash crop halophytes by a quick check system: Determination of the threshold of salinity tolerance and the ecophysiological demands. In: Cash crop halophytes: recent studies, pp. 5-17. Springer, Dordrecht.

Lailhacar, S., Hugo, R., Silva, H., Caldentey, J. 1995. Rendimiento de leña y recuperación al corte en diferentes especies y procedencias arbustivas del género Atriplex. Revista de Ciencias Forestales (Chile) 10: 85-97.

Lutts, S., Lefevre, I. 2015. How can we take advantage of halophyte properties to cope with heavy metal toxicity in salt-affected areas? Annals of Botany 115: 509-528.

Marticorena, C., Quezada, M. 1985. Catálogo de la flora vascular de Chile. Gayana Botánica 42 (1-2): 1-157.

Marticorena, C. 1990. Contribución a la estadística de la flora vascular de Chile. Gayana Botánica 47 (3-4): 85-113.

Matthei, O., Marticorena, C., Rodríguez, R. 1995. Manual de las Malezas que Crecen en Chile. Editorial Alfabeta, Chile. $545 \mathrm{pp}$.

Menzel, U., Lieth, H. 2003. Halophyte Database V 2.0 update. In: Lieth H., Mochtchenko, M. (eds.), Cash Crop Halophytes:
Recent Studies: 10 Years after Al Ain Meeting, Vol. 38 pp. 231. Springer, U.S.A.

Ministerio del Medio Ambiente. 2017. Inventario Nacional de Especies de Chile. URL: http://especies.mma.gob.cl/ CNMWeb/Web/WebCiudadana/Default.aspx. Accedido: 16 de diciembre 2017.

Moreira-Muñoz, A. 2011. Plant Geography of Chile. Springer Science \& Bussiness Media, New York, U.S.A. 343 pp.

Munns, R., Tester, M. 2008. Mechanisms of salinity tolerance. Annual Review of Plant Biology 59(1): 651-681.

O'LeARY, J., GLENN, E.P. 1994. Global distribution and potential for halophytes. In: Squires, V., Ayoub, A.T. (eds.), Halophytes as a resource for livestock and for rehabilitation of degraded lands. pp 7-18. Springer, Dordrecht.

Poblete, V., Campos, V., González, L. 1991. Anatomical leaf adaptations in vascular plants of a salt marsh in the Atacama Desert (Chile). Revista Chilena de Historia Natural 64: 65-75.

Ramírez, C., Álvarez, M. 2012. Flora y vegetación hidrófila de los Humedales Costeros de Chile. En: Fariña, J.M., Camaño, A. (eds.), Humedales costeros de Chile: Aportes científicos a su gestión sustentable, pp. 101-145. Ediciones UC, Santiago, Chile.

Ramírez, C., San Martín, C. 2006. Diversidad de macrófitos chilenos. En: Vila, I., Veloso, A., Schlatter, R., Ramírez, C. (eds.), Macrófitas e invertebrados de los sistemas líminicos de Chile. pp. 21-61. Editorial Universitaria, Santiago, Chile.

Ramírez, C., Fariña, J.M., Camaño, A., San Martín, C., Pérez, Y., Solís J.L., Valdivia, O. 2018. The case of the Itata estuary (Bio-Bio Region-Chile) plant formations: anthropogenic interference or natural disturbance-induced diversity enrichment? Mediterranean Botany 39(1): 17-34.

Rhodes, D., Felker, P. 1988. Mass screening of Prosopis (mesquite) seedlings for growth at seawater salinity concentrations. Forest Ecology and Management 24(3): 169-176.

San Martín, C., Contreras, D., San Martín, J., Ramírez, C. 1992. Vegetación de las marismas del centro-sur de Chile. Revista Chilena de Historia Natural 65: 327-342.

Santos, J., Mohammed, A.A., Aronson, J., Flowers, T.J. 2015. eHALOPH a database of salt-tolerant plants: helping put halophytes to work. Plant and Cell Physiology 57(1): e10.

Shrigel, M., Ben-Exra, D., Shauli, L., Sagi, M., Ventura, Y., Samocha, T., Lee, J.J. 2013. Constructed wetland with Salicornia as a biofilter for mariculture effluents. Aquaculture 412: 52-63.

Teillier, S. 1998. Flora y vegetación alto andina del área de Collaguasi, salar de Coposa Andes del norte de Chile. Revista Chilena de Historia Natural 71: 313-329.

Teillier, S., Becerra, P. 2003. Flora y vegetación del salar de Ascotán, Andes del norte de Chile. Gayana Botánica 60: 114-122.

Toderich, K.N., Ismail, S., Juylova, E.A., Rabbimov, A.A., Bekchanov, B.B., Shyuskaya, E.V., Gismatullina, L.G., Osamu, K., Radjabov, T.F. 2008. New approaches for biosaline agriculture development, management and conservation of sandy desert ecosystems. In: Abdelly, Ch., Öztürk, M., Ashraf, M., Grignon C. (eds.), Biosaline Agriculture and High Salinity Tolerance, pp. 247-264. Springer, Switzerland.

Zuloaga, F.O., Morrone, O., Belgrano, M.J. 2008. Catálogo de las plantas vasculares del Cono Sur (Argentina, Sur de Brasil, Chile, Paraguay y Uruguay). Missouri Botanical Garden Press, St. Louis, U.S.A. 3486 pp. 
Diversidad de especies halófitas presentes en Chile: OrREgo, F. ET AL.

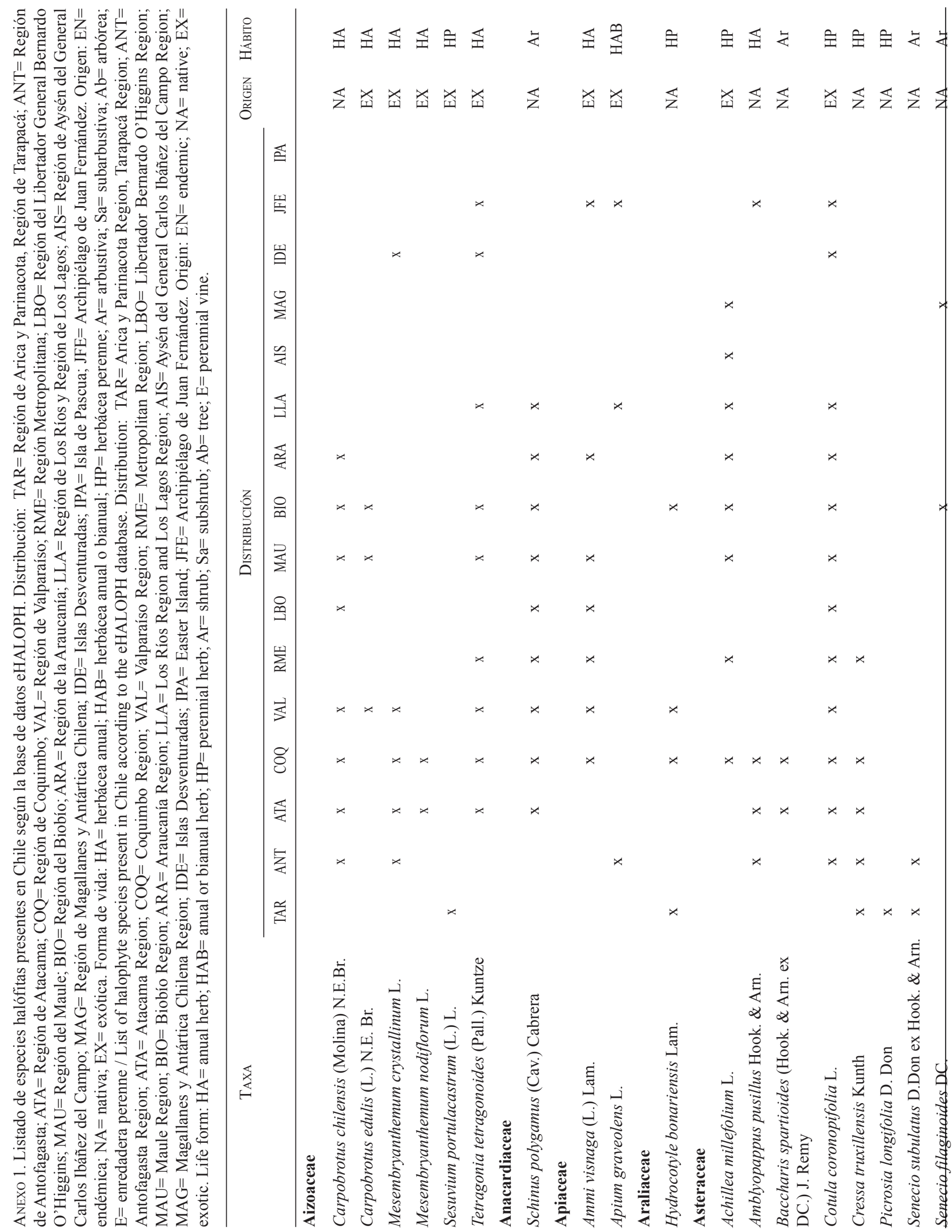




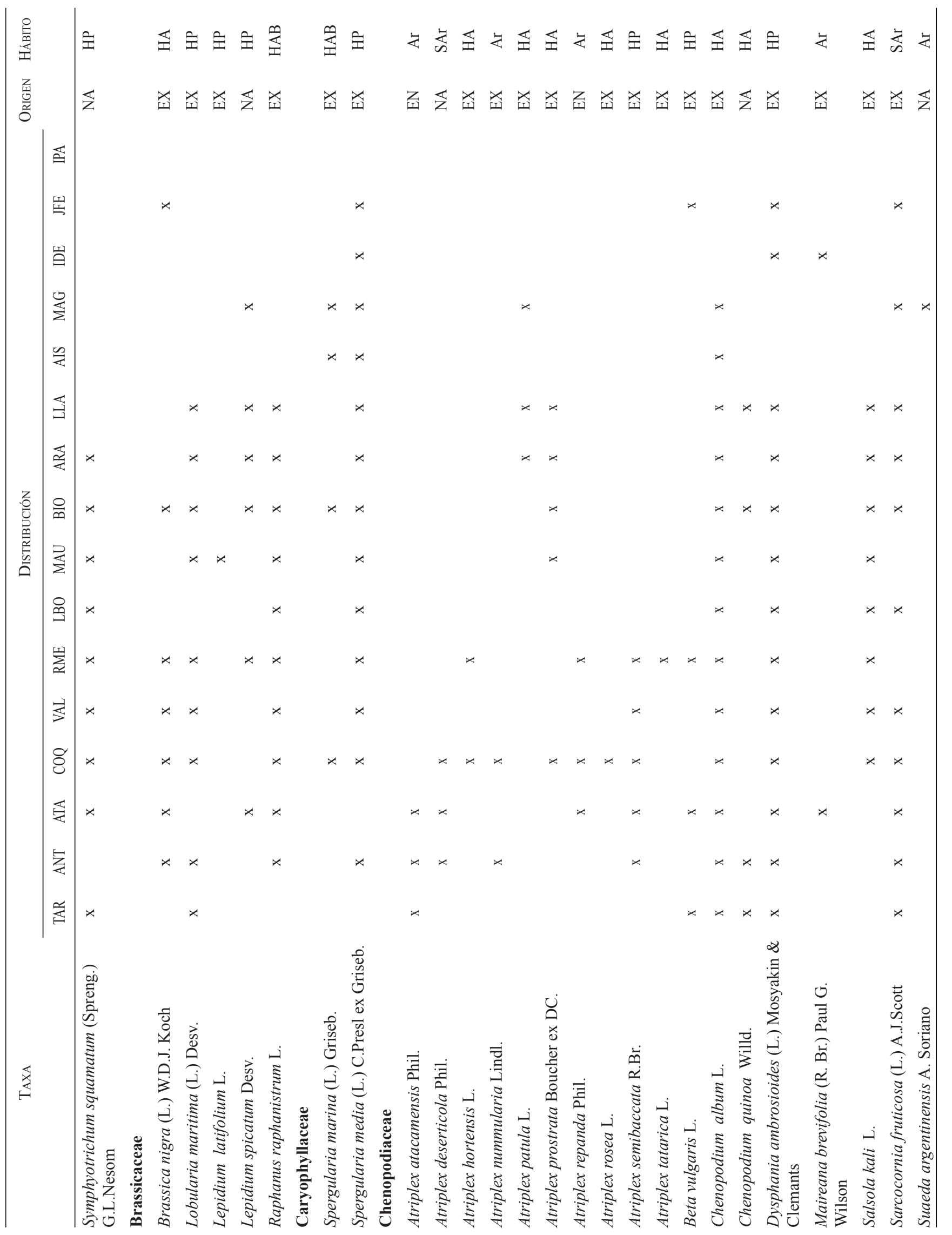


Diversidad de especies halófitas presentes en Chile: OrRego, F. ET AL.

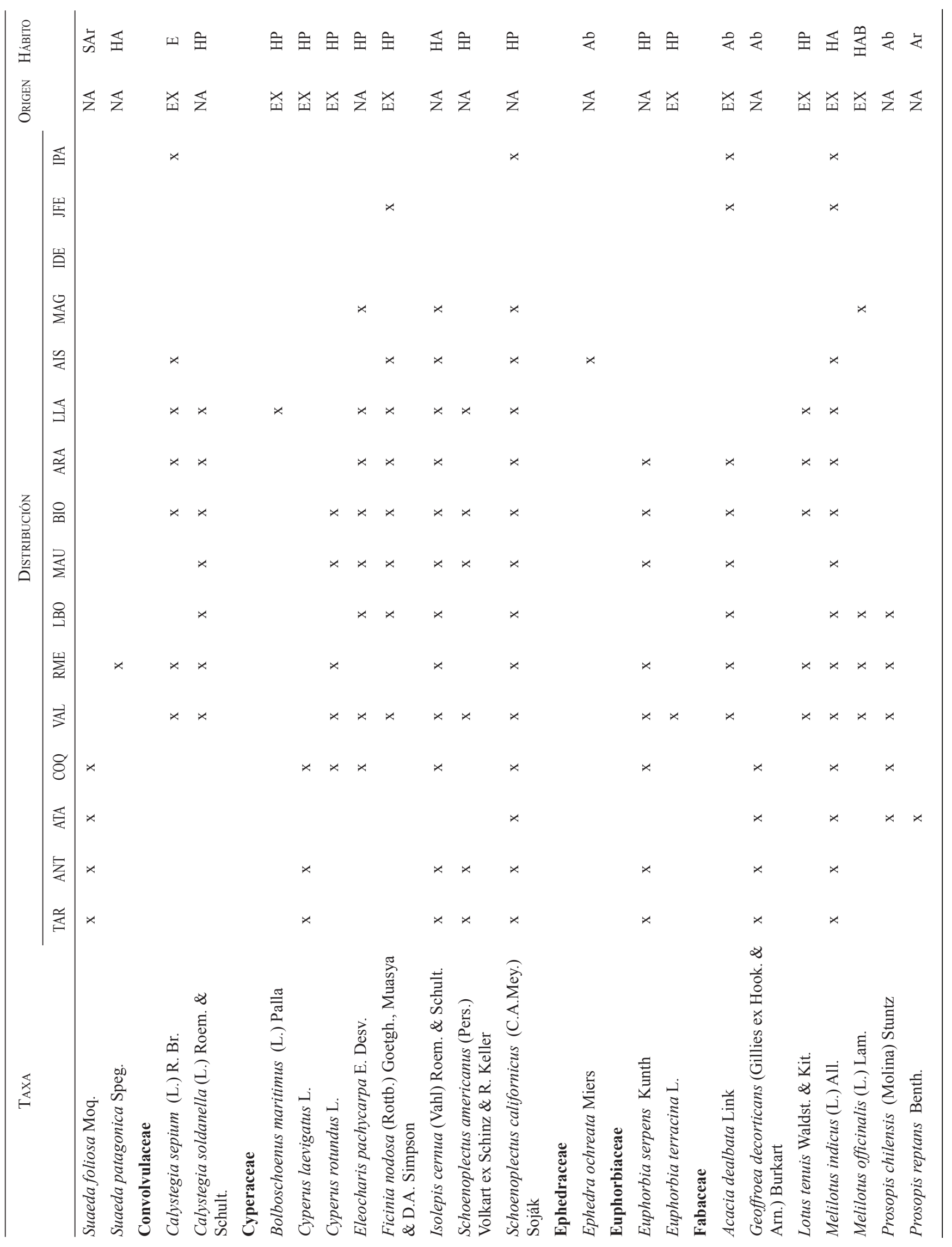




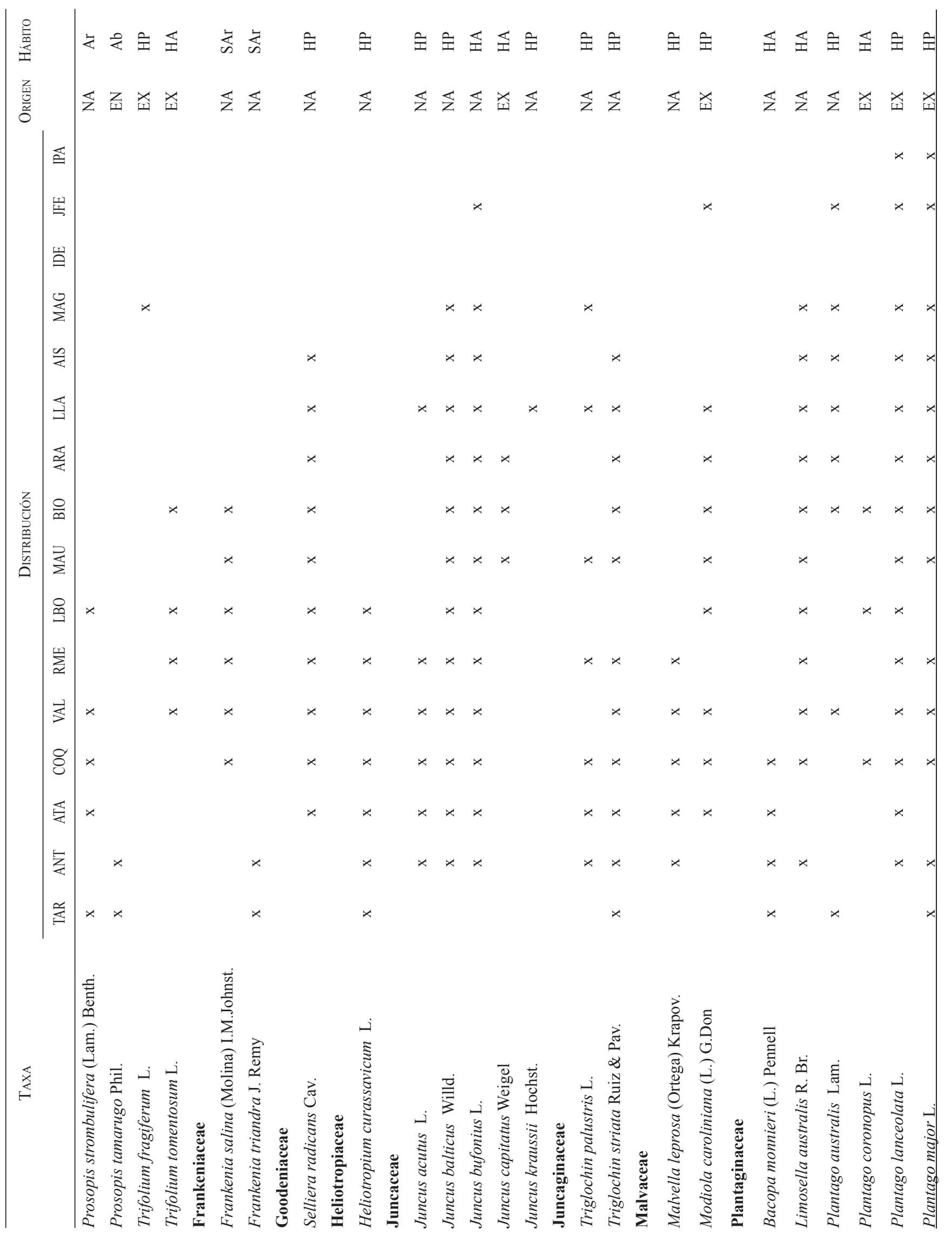




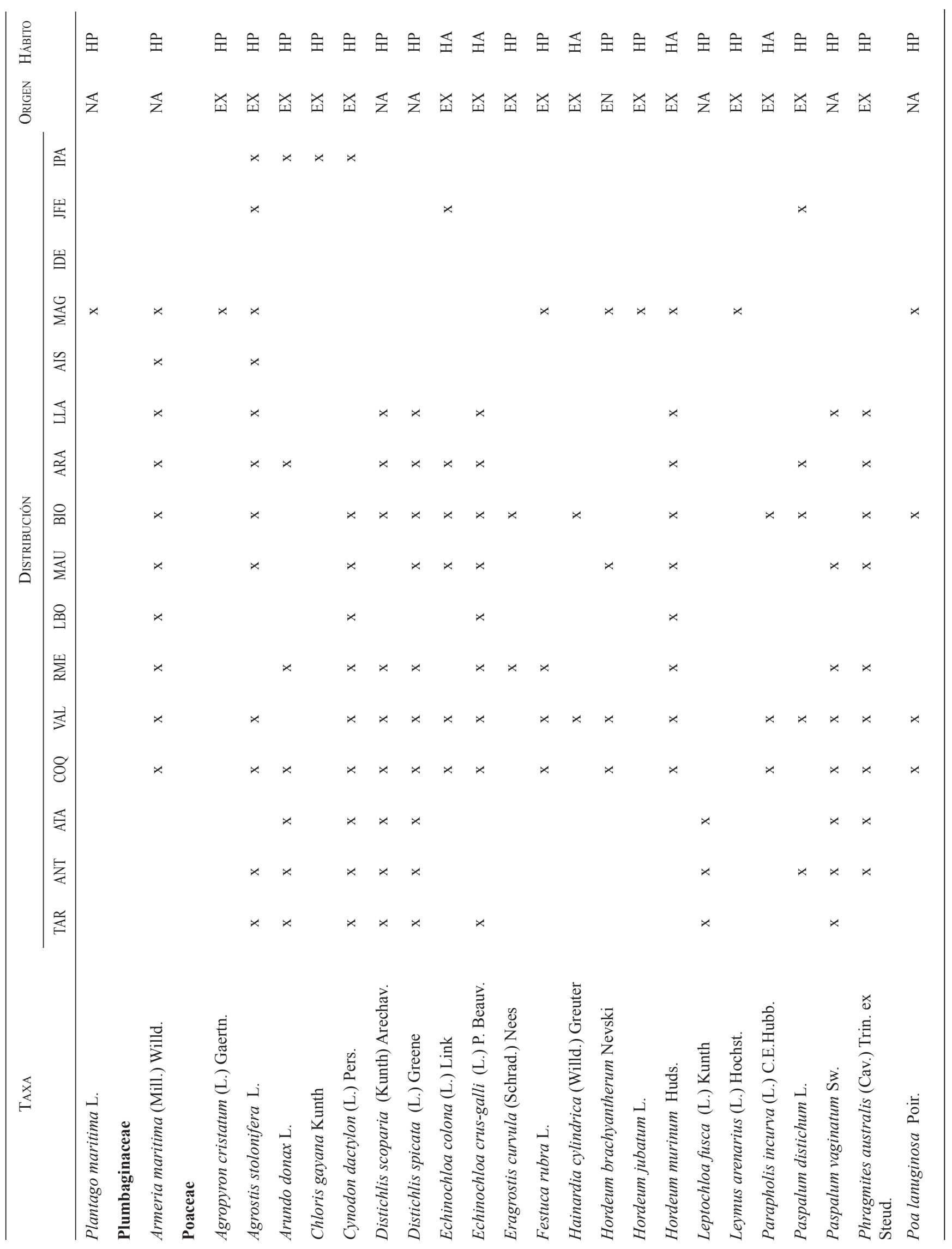




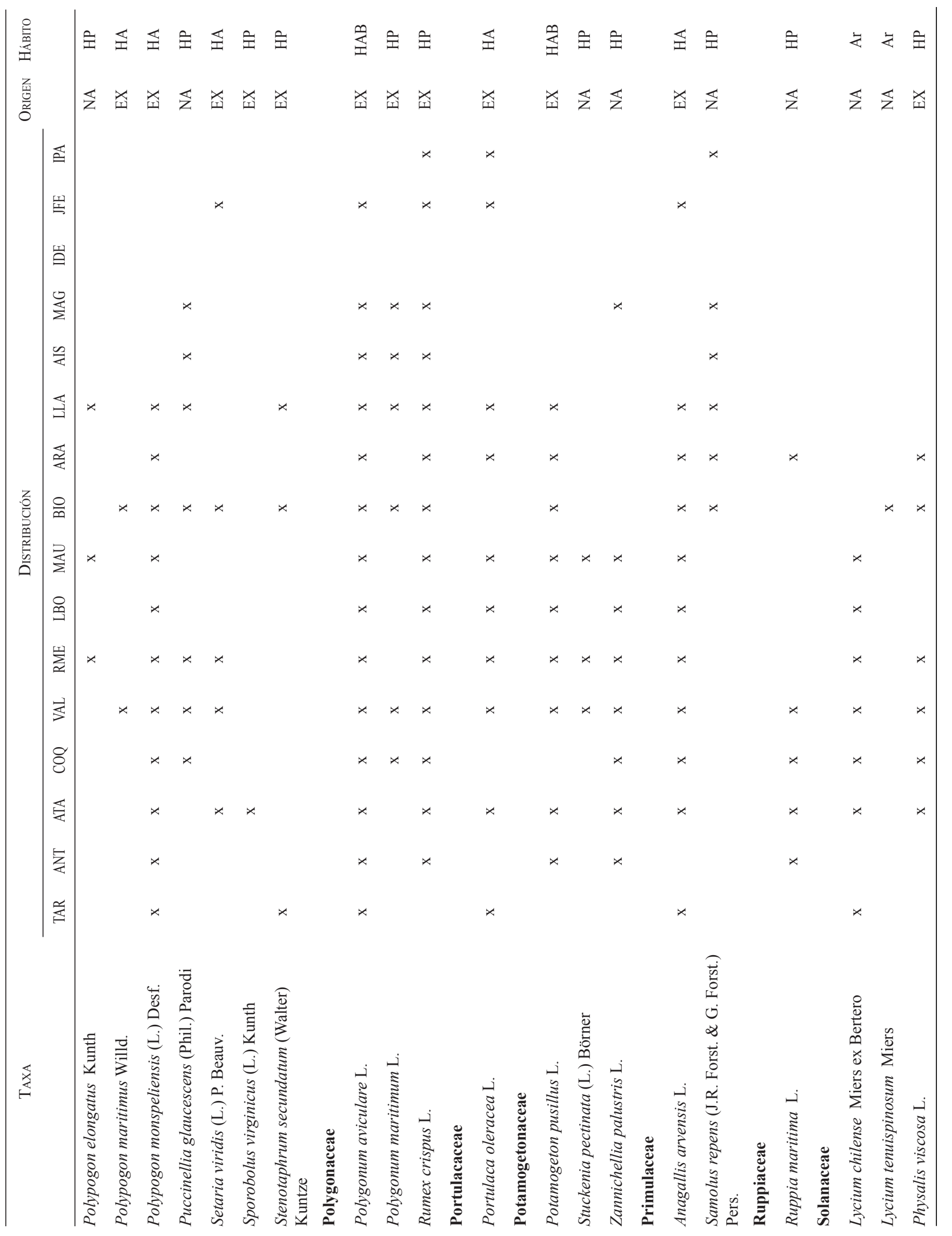


Diversidad de especies halófitas presentes en Chile: OrRego, F. ET AL.

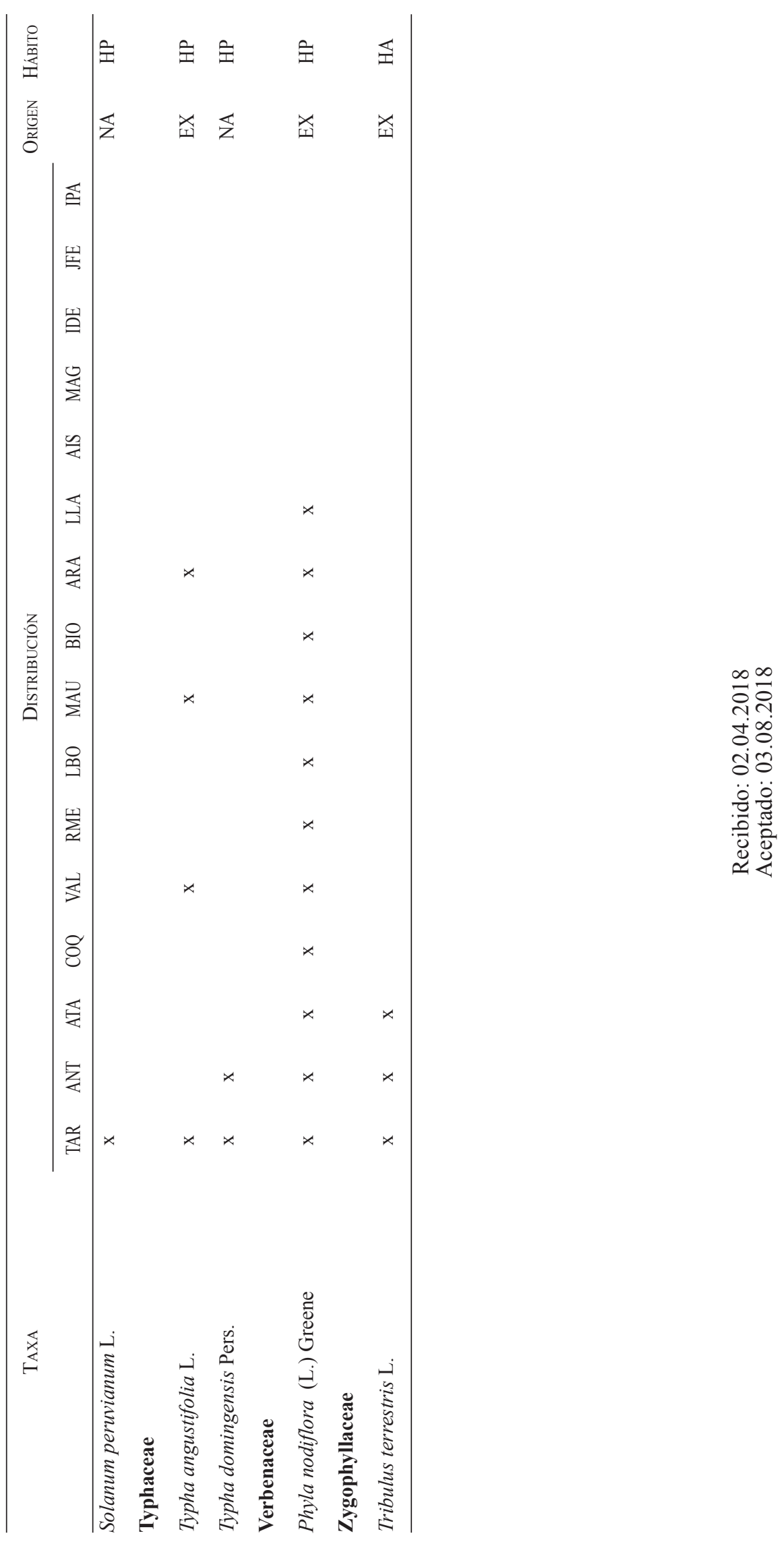

